\title{
Bioremediation of hydraulic fracturing sludge
}

\section{Biorremediación de lodos de fractura hidráulica}

\author{
Aracely Maldonado-Torres ${ }^{2}$, Carmen Sánchez ${ }^{3}$, Eduardo Osorio-Hernández ${ }^{1}$, José Alberto \\ López-Santillán ${ }^{1}$, Benigno Estrada-Drouaillet ${ }^{1 *}$. \\ 1 Posgrado e Investigación. Facultad de Ingeniería y Ciencias. Universidad Autónoma de \\ Tamaulipas. Centro Universitario Victoria, C.P. 87000, Cd. Victoria, Tamaulipas, México. \\ ${ }^{2}$ Doctorado en Ciencias Sistemas Agropecuarios y Medio Ambiente. Facultad de Ingeniería y \\ Ciencias. Universidad Autónoma de Tamaulipas. Centro Universitario Victoria, C.P. 87000, Cd. \\ Victoria, Tamaulipas, México. \\ ${ }^{3}$ Centro de Investigaciones en Ciencias Biológicas. Universidad Autónoma de Tlaxcala. Km 10.5 \\ carretera San Martin Texmelucan - Tlaxcala, Ixtacuixtla de Mariano Matamoros, C.P. 90120, Villa \\ Mariano Matamoros, Tlaxcala, México. \\ *benestrada@uat.edu.mx \\ https://doi.org/10.29267/mxjb.2016.1.2.29
}

\begin{abstract}
Hydraulic fracturing is an unconventional technique that has been used since the early nineties for the extraction of hydrocarbons, such as natural gas, located in sedimentary deposits of shale rocks or shales, for which it isn't possible the extraction by conventional methods. However, although the hydraulic fracturing allows the use of these resources, has created controversy about the use of this technique, mainly because of the waste generated during the process. Such is the case of the residual sludge obtained in the backflow produced by hydraulic fracturing; in which hydrocarbons, potentially toxic elements and isotopes gamma have been founds, which are harmful to the environment and human health. For this reason it is necessary to find remediation techniques that mitigate these effects. In this article the main techniques of bioremediation are discussed as alternatives to treat the sludge generated during the process of hydraulic fracturing
\end{abstract}

Keywords: bioremediation, natural gas, sludge.

\section{RESUMEN}

La fractura hidráulica es una técnica no convencional que se ha utilizado desde inicios de los años noventa para la extracción de hidrocarburos, como el gas natural, localizados en depósitos sedimentarios de rocas lutita o esquistos, para los cuales no es posible la extracción mediante métodos convencionales. Sin embargo, aunque la fractura hidráulica permite el aprovechamiento de estos recursos, se ha creado una polémica en relación con el uso de esta técnica, debido principalmente a los residuos que genera durante el proceso. Tal es el caso de los lodos residuales que se obtienen del flujo de retorno producto de la fractura hidráulica; en los cuales se han encontrado hidrocarburos, elementos potencialmente tóxicos e isotopos gamma, los cuales son nocivos para el ambiente y la 
salud humana. Por tal motivo es necesario encontrar técnicas de remediación que atenúen estos efectos. En este artículo se abordan las principales técnicas de biorremediación como alternativas para tratar los lodos generados durante el proceso de fractura hidráulica. Palabras clave: biorremediación, gas natural, lodos.

\section{INTRODUCCIÓN}

El gas natural es una mezcla de hidrocarburos simples conformada principalmente por alcanos de bajo peso molecular, de los cuales alrededor del $95 \%$ está constituido por metano y en orden decreciente por otros alcanos, cuanto más compleja su estructura menor es su concentración (Hart, 2013), por su origen contiene también impurezas como nitrógeno, dióxido de carbono, sulfuro de hidrógeno, vapor de agua y radón (Secretaría de Energía, 2014). De forma natural este hidrocarburo puede encontrarse asociado con el petróleo o en forma de gas no asociado en formaciones geológicas conocidas como lutitas, shale, pizarra o esquisto por lo que comercialmente también se le conoce con estos nombres. Las formaciones tipo shale son impermeables, altamente porosas y se encuentran a profundidades de más de $2 \mathrm{~km}$ bajo la superficie terrestre (Bjørlykke, 2010; Hart, 2013; Ma et al., 2013, Wei et al., 2013; Wang et al., 2015), por lo que es necesario recurrir a técnicas no convencionales como la fractura hidráulica y la perforación horizontal para explotarlas (Wheeler et al., 2015; Wang et al., 2015; Chen et al., 2015); sin embargo, al ser una fuente abundante y eficiente de energía es considerado como una fuente alterna de energía para el futuro (Chen et al., 2012; Ryzhov et al., 2012). La fractura hidráulica ha incrementado la capacidad de extracción del gas shale por lo que se le considera clave tecnológica para su desarrollo (Zhang et al., 2016; Wang et al., 2015; Chen et al., 2015), al grado de generar una producción de alrededor de $206,685 \times 10^{12} \mathrm{~m}^{3}$ anuales a nivel mundial (Fig. 1).

Esta técnica consiste en inyectar a altas presiones una mezcla de fluidos (agua, arena y compuestos químicos, apuntalantes o aditivos (Tabla 1), que tienen la finalidad de fracturar las vetas de gas natural, mantenerlas abiertas y de esta forma provocar la salida del recurso hacia la superficie donde se almacena en pozos excavados previamente (Bjørlykke, 2010; Hart, 2013; Moreu, 2013; Zhang et al., 2016; Wheeler et al., 2015), de este proceso también se obtienen aguas de reflujo y lodos residuales que contienen niveles altos de sólidos totales disueltos, compuestos tóxicos de origen natural como materiales radiactivos disueltos procedentes de las formaciones de lutitas (Zhang et al., 2016; EPA, 2015; Fair, 2014). Se estima que por cada pozo excavado mediante fractura hidráulica se generan entre 499 y $1078 \mathrm{~m}^{3}$ de lodos residuales (IEA, 2012; Jiang et al., 2014). Estos lodos junto con los fluidos de retorno se almacenan en embalses superficiales de 100 x $100 \mathrm{~m}$ de superficie (IEA, 2012) (Fig. 2), que corren el riesgo de dañarse con el tiempo y provocar desgarres por los que se vierte su contenido al suelo de los alrededores ocasionando la contaminación del mismo y en consecuencia afectaciones a su estructura y fertilidad que se traducen en la reducción de su capacidad para soportar el crecimiento de las plantas y microbiota (Adams et al., 2008; Fair, 2014; Adams et al., 2014; Moubasher et al., 2015). Además, estos lodos contienen elementos altamente tóxicos y recalcitrantes que pueden integrarse a la cadena trófica donde se acumulan y biomagnifican provocando efectos adversos a la salud humana (Adams et al., 2015; Zhang et al., 2016; EPA, 2015). 


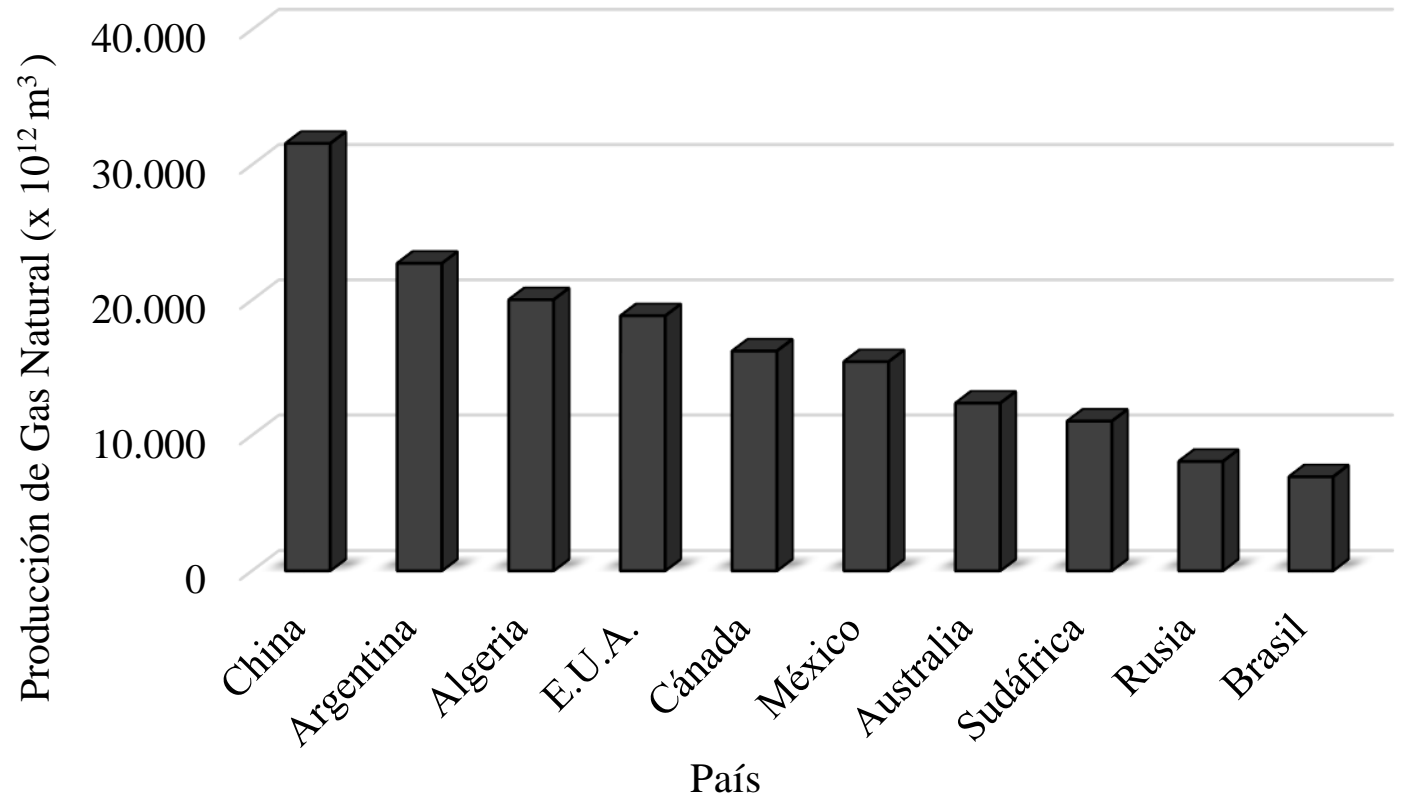

Fig. 1. Principales 10 países con recursos no probados de gas natural de lutitas técnicamente recuperables

Fuente: Elaboración propia basado en los datos publicados por https://www.eia.gov/analysis/studies/worldshalegas/

Tabla 1. Constituyentes típicos de los fluidos de fractura hidráulica, composición volumétrica, propósito y ejemplos con número CAS (Chemical Abstract Service).

\begin{tabular}{|c|c|c|c|c|}
\hline Constituyente & Volumen (\%) & Propósito & Nombre químico & CAS \\
\hline Agua y arena & 99.50 & $\begin{array}{c}\text { Granos de arena apuntalantes que } \\
\text { mantienen abiertas las microfracturas }\end{array}$ & & $007647-01-0$ \\
\hline Ácido & 0.123 & $\begin{array}{c}\text { Ayuda a disolver los minerales e inicia } \\
\text { las grietas en la roca }\end{array}$ & Ácido clorhídrico & $007647-01-0$ \\
\hline \multirow{5}{*}{$\begin{array}{l}\text { Reductor de } \\
\text { fricción }\end{array}$} & \multirow{5}{*}{0.088} & \multirow{5}{*}{$\begin{array}{l}\text { Se adhiere al agua para minimizar la } \\
\text { fricción entre el fluido y la tubería. } \\
\text { Fluido portador de poliacrilamida } \\
\text { reductor de fricción }\end{array}$} & Poliacrilamida & 009003-05-8 \\
\hline & & & Destilado de petróleo & 064741-85-1 \\
\hline & & & $\begin{array}{c}\text { Destilado ligero de petróleo } \\
\text { tratado con } \mathrm{H}_{2}\end{array}$ & $064742-47-8$ \\
\hline & & & Metanol & 000067-56-1 \\
\hline & & & Etilenglicol & $000107-21-1$ \\
\hline
\end{tabular}




\begin{tabular}{|c|c|c|c|c|}
\hline Constituyente & Volumen (\%) & Propósito & Nombre químico & CAS \\
\hline \multirow{6}{*}{ Surfactante } & \multirow{6}{*}{0.085} & \multirow{6}{*}{$\begin{array}{l}\text { Aumenta la viscosidad del fluido de } \\
\text { fracturación, estabilizador y fluido } \\
\text { portador para el invierno para los } \\
\text { ingredientes activos de los } \\
\text { tensoactivos }\end{array}$} & Laurilsulfato & $000151-21-3$ \\
\hline & & & Etanol & $000064-17-5$ \\
\hline & & & Naftalina & $000091-20-3$ \\
\hline & & & Metanol & $000067-56-1$ \\
\hline & & & Alcohol isopropílico & 000067-63-0 \\
\hline & & & 2-butoxietanol & $000111-76-2$ \\
\hline Sal & 0.06 & Crea un fluido portador de salmuera & & \\
\hline \multirow{3}{*}{$\begin{array}{l}\text { Inhibidor de } \\
\text { escala }\end{array}$} & \multirow{3}{*}{0.043} & \multirow{3}{*}{$\begin{array}{l}\text { Previene las incrustaciones de cal en } \\
\text { las tuberías }\end{array}$} & $\begin{array}{l}\text { Copolímero de acrilamida y } \\
\text { acrilato de sodio }\end{array}$ & $025987-30-8$ \\
\hline & & & Policarboxilato de sodio & NA \\
\hline & & & Sal de ácido fosfórico & NA \\
\hline \multirow{5}{*}{$\begin{array}{l}\text { Agente de } \\
\text { ajuste - pH }\end{array}$} & \multirow{5}{*}{0.011} & \multirow{5}{*}{$\begin{array}{l}\text { Mantiene la eficacia de aditivos } \\
\text { químicos y otros componentes como } \\
\text { agentes de reticulación ajustando el } \\
\text { pH de fluido }\end{array}$} & Hidróxido de sodio & $001310-73-2$ \\
\hline & & & Hidróxido de potasio & $001310-58-3$ \\
\hline & & & Ácido acético & 000064-19-7 \\
\hline & & & Carbonato de sodio & 000497-19-8 \\
\hline & & & Carbonato de potasio & 000584-08-7 \\
\hline \multirow{4}{*}{$\begin{array}{l}\text { Control de } \\
\text { hierro }\end{array}$} & \multirow{4}{*}{0.004} & \multirow{4}{*}{$\begin{array}{l}\text { Evita la precipitación de óxidos de } \\
\text { metales }\end{array}$} & Ácido cítrico & $000077-92-9$ \\
\hline & & & Ácido acético & 000064-19-7 \\
\hline & & & Ácido tioglicólico & 000068-11-1 \\
\hline & & & Eritorbato de sodio & $006381-77-7$ \\
\hline \multirow{4}{*}{$\begin{array}{l}\text { Inhibidor de } \\
\text { corrosión }\end{array}$} & \multirow{4}{*}{0.002} & \multirow{4}{*}{$\begin{array}{l}\text { Prevenir la corrosión de tuberías, } \\
\text { estabilizador y / o agente de } \\
\text { acondicionamiento para el invierno }\end{array}$} & Isopropanol & 000067-63-0 \\
\hline & & & Metanol & $000067-56-1$ \\
\hline & & & Ácido fórmico & 000064-18-6 \\
\hline & & & Acetaldehído & $000075-07-0$ \\
\hline \multirow{3}{*}{ Biocida } & \multirow{3}{*}{0.001} & \multirow{3}{*}{$\begin{array}{c}\text { Elimina o minimiza el crecimiento de } \\
\text { bacterias tóxicas en el agua que } \\
\text { produce subproductos corrosivos }\end{array}$} & Glutaraldehído & $000111-30-8$ \\
\hline & & & $\begin{array}{c}\text { Cloruro de amonio } \\
\text { cuaternario }\end{array}$ & $012125-02-9$ \\
\hline & & & $\begin{array}{c}\text { Tetra sulfato de } \\
\text { hidroximetil-fosfonio }\end{array}$ & $055566-30-8$ \\
\hline \multirow{5}{*}{ Fracturador } & \multirow{17}{*}{0.083} & \multirow{5}{*}{$\begin{array}{l}\text { Permite una rotura retardada abajo del } \\
\text { gel }\end{array}$} & Persulfato de amonio & $007727-54-0$ \\
\hline & & & Cloruro de sodio & 007647-14-5 \\
\hline & & & Peróxido de magnesio & $014452-57-4$ \\
\hline & & & Óxido de magnesio & 001309-48-1 \\
\hline & & & Cloruro de calcio & 010043-52-4 \\
\hline \multirow{3}{*}{$\begin{array}{l}\text { Estabilizador } \\
\text { de arcilla }\end{array}$} & & \multirow{3}{*}{$\begin{array}{l}\text { Previene el paso de las arcillas de la } \\
\text { hinchazón o su paso }\end{array}$} & Cloruro de colina & $000067-48-1$ \\
\hline & & & $\begin{array}{c}\text { Tetrametilo de cloruro de } \\
\text { amonio } \\
\end{array}$ & $000075-57-0$ \\
\hline & & & Cloruro de sodio & $007647-14-5$ \\
\hline \multirow{9}{*}{ Reticulante } & & \multirow{9}{*}{$\begin{array}{l}\text { Mantiene la viscosidad del fluido a } \\
\text { medida que aumenta la temperatura, } \\
\text { fluido portador de borato o reticulador } \\
\text { de circonato }\end{array}$} & Destilado de petróleo & $064741-85-1$ \\
\hline & & & $\begin{array}{l}\text { Tratada del destilado de } \\
\text { petróleo ligero con } \mathrm{H}_{2}\end{array}$ & $064742-47-8$ \\
\hline & & & Metaborato de potasio & 013709-94-9 \\
\hline & & & Circonato de trietanolamina & 101033-44-7 \\
\hline & & & Tetraborato de sodio & 001303-96-4 \\
\hline & & & Ácido bórico & $001333-73-9$ \\
\hline & & & Complejo de zirconio & 113184-20-6 \\
\hline & & & Sales de borato & NA \\
\hline & & & Etilenglicol & 000107-21-1 \\
\hline
\end{tabular}




\begin{tabular}{|c|c|c|c|c|}
\hline Constituyente & Volumen $(\%)$ & Propósito & Nombre químico & CAS \\
\hline \multirow{4}{*}{$\begin{array}{l}\text { No - } \\
\text { emulsionante }\end{array}$} & & \multirow{4}{*}{$\begin{array}{l}\text { Previene la formación de emulsiones } \\
\text { en el fluido de fractura }\end{array}$} & Metanol & 000067-56-1 \\
\hline & & & Laurilsulfato & 000151-21-3 \\
\hline & & & Isopropanol & $000067-63-0$ \\
\hline & & & Etilenglicol & 000107-21-1 \\
\hline \multirow{6}{*}{$\begin{array}{l}\text { Agente } \\
\text { gelificante }\end{array}$} & & \multirow{6}{*}{$\begin{array}{c}\text { Espesa el agua con el fin de suspender } \\
\text { la arena, fluido portador para geles } \\
\text { líquidos de goma guar }\end{array}$} & Goma de guar & 009000-30-0 \\
\hline & & & Destilado de petróleo & $064741-85-1$ \\
\hline & & & $\begin{array}{l}\text { Destilado de petróleo ligero } \\
\text { tratado con } \mathrm{H}_{2}\end{array}$ & $064742-47-8$ \\
\hline & & & Metanol & 000067-56-1 \\
\hline & & & Mezcla de polisacáridos & 068130-15-4 \\
\hline & & & Etilenglicol & 000107-21-1 \\
\hline
\end{tabular}

Fuente: http://fracfocus.org/chemical-use/what-chemicals-are-used y Gregory et al., 2011

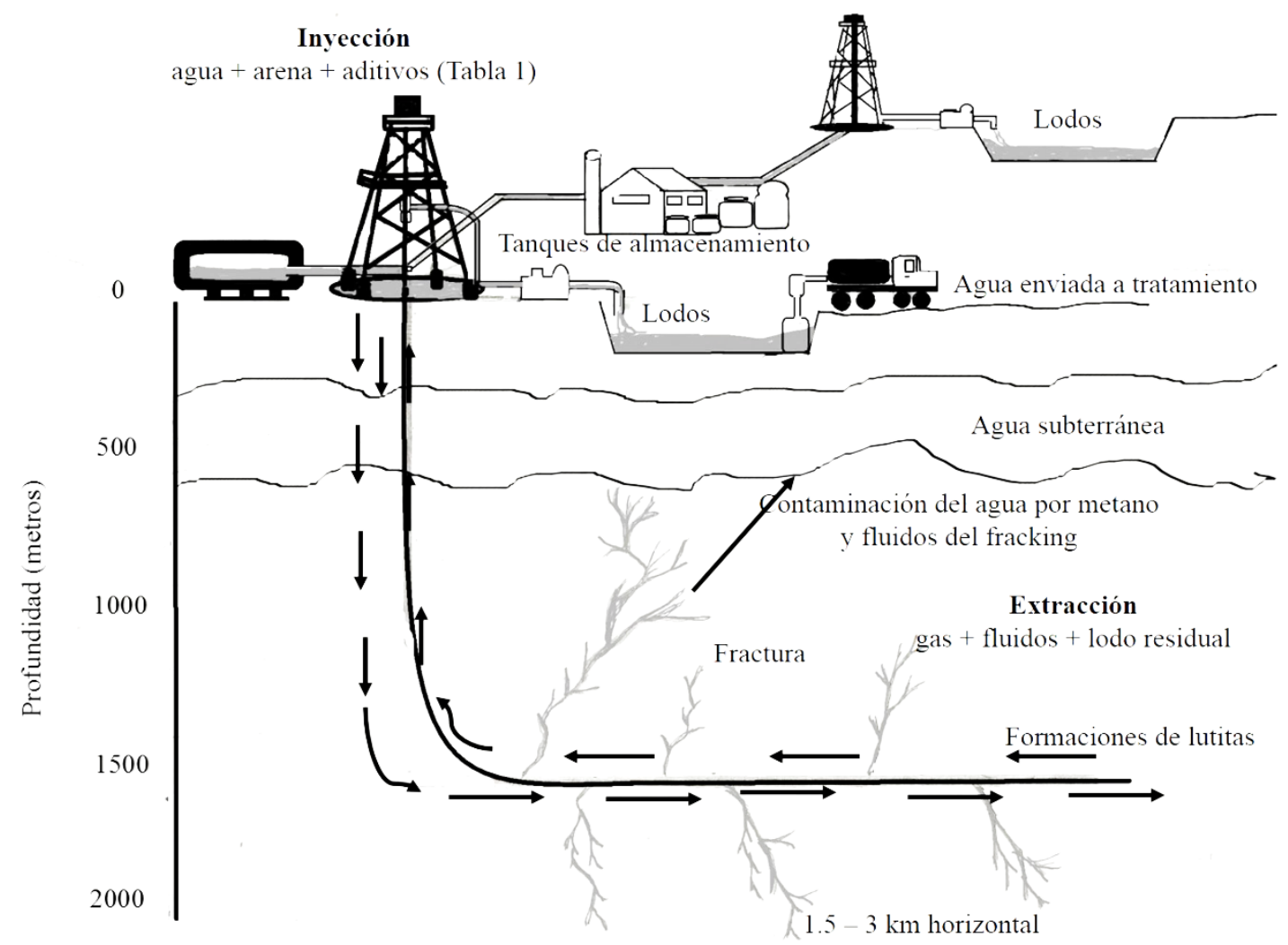

Fig. 2. Proceso de fractura hidráulica y generación de lodos residuales. 


\section{LODOS RESIDUALES}

Los lodos residuales generados durante las actividades petroleras como la extracción de gas natural son catalogados a nivel mundial como residuos peligrosos (SEMARNAT, 2006; Ning et al., 2009; EPA, 2015), debido a que contienen entre otros contaminantes sales, metales, hidrocarburos y radionúclidos (Fair, 2014; Zhang et al., 2016). Se estima que por cada pozo explotado por fractura hidráulica se obtienen $1.41 \times 10^{8} \mathrm{~m}^{3}$ de gas natural y para la fracturación se emplean $15 \times 10^{3} \mathrm{~m}^{3}$ de agua, de los cuales se recuperan aproximadamente $9,495 \mathrm{~m}^{3}$, generándose en el proceso entre 499 y $1078 \mathrm{~m}^{3}$ de lodo residual por pozo excavado (IEA, 2012; Jiang et al., 2014); si éstos lodos no reciben un tratamiento adecuado y disposición pueden afectar tanto al ambiente como a la salud humana (EPA, 2015). La generación estimada de estos residuos para las cuencas localizadas en México con base a las reservas no probadas de gas natural se puede apreciar en la Tabla 2.

Tabla 2. Generación estimada de lodo residual para México con base en las reservas estimadas de gas natural técnicamente recuperable por fractura hidráulica.

\begin{tabular}{ccc}
\hline Cuenca & $\begin{array}{c}\text { Reservas estimadas de gas natural } \\
\text { técnicamente recuperable }\left(\mathrm{m}^{3}\right)\end{array}$ & $\begin{array}{c}\text { Rango de generación de lodo } \\
\text { residual estimado }\left(\mathrm{m}^{3}\right)\end{array}$ \\
\hline Burgos & $1.11 \times 10^{13}$ & $3.94 \times 10^{7}-7.89 \times 10^{7}$ \\
Sabinas & $3.51 \times 10^{12}$ & $1.24 \times 10^{7}-2.49 \times 10^{7}$ \\
Tampico & $6.51 \times 10^{11}$ & $2.31 \times 10^{6}-4.62 \times 10^{6}$ \\
Tuxpan & $5.66 \times 10^{10}$ & $2.01 \times 10^{5}-4.01 \times 10^{5}$ \\
Veracruz & $8.49 \times 10^{10}$ & $3.01 \times 10^{5}-6.02 \times 10^{5}$ \\
Total & $1.54 \times 10^{13}$ & $5.47 \times 10^{7}-1.09 \times 10^{8}$ \\
\hline
\end{tabular}

Fuente: Elaboración propia a partir de IEA, 2014; IEA, 2013 y Jiang et al., 2014).

\subsection{Composición de los lodos residuales}

Dependiendo de la actividad que origina a los lodos residuales están compuestos por una amplia variedad de sustancias; en lodos provenientes del procesamiento de gas natural se han encontrado hidrocarburos que alcanzan concentraciones de hasta $334,766 \mathrm{mg} \mathrm{kg}^{-1}$, además de compuestos de entre 6 y 73 átomos de carbono en su estructura, cromo, zinc, fierro, plomo, vanadio, cadmio, níquel, cobre, magnesio, fósforo y potasio (CastorenaCortés et al., 2009).

En lodos de perforación se han detectado sulfuros, arsénico, bario, berilio, cobalto, cromo, molibdeno, níquel, plomo, selenio, vanadio, zinc, además de compuestos BTEX que corresponden a benceno, etilbenceno, metil-butileter, tolueno y xileno, compuestos orgánicos volátiles como freon-11, acetona, 2-butanona, benceno, tolueno, 1,1,1,2tetracloroetano, etilbenceno, m\&p-xileno, o-xileno, isopropilbenceno, 1,3,5- 
trimetilbenceno, 1,2,4-trimetilbenceno, sec-butilbenceno, naftaleno y compuestos orgánicos semivolátiles en particular, si-n-octilftalato, naftaleno, fluoreno, fenantreno y 2-metil naftaleno (Zagrean, 2002).

Los lodos procedentes del proceso de fractura hidráulica reflejan las características de la formación de donde fueron drenados y la composición de los fluidos utilizados (Tabla 1), se componen de sólidos disueltos precipitados del agua de proceso y que contienen por lo general aceites, material suelto con compuestos de silica y grandes cantidades de bario, pues un aditivo común es la barita que se emplea como agente de ponderación para mejorar la viscosidad del fluido y su capacidad para contrarrestar la presión de formación y para flotar material de suelo a la superficie, además la perforación convencional genera anualmente $141.5 \times 10^{3} \mathrm{~m}^{3}$ de lodos considerados TENORM (Technologically Enhanced Naturally Occurring Radioactive Material) en los cuales se puede encontrar Ra-226 en concentraciones promedio de 75 Picocuries por gramo $\left(\mathrm{pCi} \mathrm{g}^{-1}\right)$ y $\mathrm{Pb}-210$ que puede llegar a concentraciones de $27 \times 10^{3} \mathrm{pCi} \mathrm{g}^{-1}$ (EPA, 2015).

\section{PROCESOS DE BIORREMEDIACIÓN EMPLEADOS EN LODOS RESIDUALES DE FRACTURA HIDRAÚLICA}

La biorremediación es una de las técnicas más usadas para recuperar sustratos contaminados consiste en utilizar organismos capaces de vivir en sitios que contienen compuestos tóxicos, por ejemplo, en el caso de hidrocarburos estos pueden ser utilizados como fuente de carbono (Bewley et al., 2001; Bundy et al., 2002; Castorena-Cortés et al., 2009; Adams et al., 2015; Gonçalves et al., 2015), o degradarse a productos inocuos mediante el metabolismo microbiano (USEPA; 2012; Adams et al., 2015); esta técnica es ampliamente utilizada por su simplicidad, aplicabilidad en áreas extensas y la naturaleza completamente destructiva respecto a los residuos tratados. Estudios previos demuestran que puede ser una tecnología económica, eficiente y limpia para tratar lodos y sedimentos que contienen hidrocarburos biodegradables (Mrayyan \& Battikhi, 2005), además los métodos empleados en la biorremediación de suelos pueden ser adaptados para la remediación de lodos generados durante el proceso de fractura hidráulica (Castorena-Cortés et al., 2009). Los métodos más comunes en la biorremediación son la atenuación natural, bioestimulación, bioventeo, bioaumentación, biolabranza, composteo y fitorremediación (Devi et al., 2011). Es importante tener en cuenta que los parámetros que definen la eficiencia de estas técnicas son la temperatura, $\mathrm{pH}$, nutrimentos y disponibilidad de oxígeno, rangos de carbono-nitrógeno y carbono-fósforo, humedad, cantidad y estructura del contaminante (Semple et al., 2001; Bewley et al., 2001; Bundy et al., 2002; CastorenaCortés et al., 2009) y se considera que las bacterias son microorganismos esenciales para el proceso de biorremediación (Castorena-Cortés et al., 2009).

\subsection{Bioestimulación}

Las comunidades microbianas que han sido expuestas a hidrocarburos exhiben enriquecimiento selectivo y cambios genéticos que les permiten responder de forma eficiente a los derrames de estas sustancias con rangos de degradación más altos que las comunidades que no tienen antecedentes con esos contaminantes orgánicos (Atlas \& Bartha, 1988; Leahy \& Colvwell, 1990;), sin embargo, la biodegradación de hidrocarburos 
presentes en suelos puede verse limitada por la falta de nutrientes (Bundy et al., 2002). La bioestimulación consiste en la incorporación de nutrimentos que mejoren las condiciones del sustrato para la microbiota nativa que tiene la capacidad de responder de forma natural al enriquecimiento mineral cuando se encuentran en condiciones favorables aprovechando el hidrocarburo como fuente de carbono incorporándolo a su metabolismo. Los nutrientes pueden ser naturales o sintéticos, orgánicos o inorgánicos, en algunos casos se añaden enzimas que pueden estimular los procesos de remediación (Adams et al., 2015), además mediante esta aplicación se pueden obtener aceptores de electrones como fósforo, nitrógeno, oxígeno y carbono que permiten aumentar la actividad de los microorganismos degradadores (Perfumo et al., 2007); la bioestimulación también incluye los procesos de aireación, control de $\mathrm{pH}$ (el cual debe estar entre 6 y 8), temperatura y humedad la cual debe mantenerse entre el $12-25 \%$ para favorecer el crecimiento y proliferación de los microorganismos (Margesin et al., 2001; Mukherjee et al., 2005; ICSS, 2006; Adams et al., 2015).

La degradación de hidrocarburos mediante biorremediación se puede ver limitada por el contacto entre el contaminante y el microorganismo, al respecto la adición de surfactantes o tensioactivos incrementa la tensión interfacial y la partición de fase del compuesto hidrófobo, favoreciendo la degradación del contaminante (Castorena-Cortés et al., 2009). El principal desafío es que los nutrimentos añadidos se encuentren disponibles para los microorganismos y esto dependerá en gran medida del tipo de suelo existente, ya que la adición de nutrimentos puede promover el crecimiento de microorganismos heterótrofos que no realizan la degradación de hidrocarburos generando una competencia por los nutrientes (Adams et al., 2014; Adams et al., 2015). Aunado a esto es importante considerar que la mayoría de los contaminantes orgánicos son mezclas complejas como en el caso del petróleo y que la mayoría de los microorganismos metabolizan solo un rango limitado de los mismos, por lo que en estos casos es necesaria una mezcla de diferentes grupos bacterianos o consorcios para degradar una gama más amplia de hidrocarburos (Bordenave, 2007; Al-Saleh, 2009). Por otra parte la concentración del contaminante influye directamente en la actividad microbiana, una alta concentración puede tener efectos tóxicos en los microorganismos y concentraciones muy bajas pueden impedir la inducción de enzimas de degradación bacteriana (Mukherjee et al., 2005; ICSS, 2006; Adams et al., 2015).

Pese a las limitaciones mencionadas esta técnica se considera eficiente para degradar hidrocarburos totales de petróleo (HTP) (Margesin et al., 2001; Hernández et al., 2003; Olguín et al., 2007; Thompson et al., 2008; Sabate et al., 2009; Vásquez et al., 2010; Devi et al., 2011) alcanzando porcentajes de degradación superiores al $20 \%$ en períodos de 30 días (Castorena-Cortés et al., 2009; Devi et al., 2011); y recientemente en la degradación de lodos contaminados con fluidos de fractura hidráulica removiendo por encima del $50 \%$ del contaminante en tiempos de 6 a 14 días (Kekacs et al., 2015; Strong et al., 2014).

\subsection{Bioaumentación}

La bioaumentación consiste en adicionar microorganismos al sustrato cuando la microflora autóctona es insuficiente en número, capacidad degradadora o se encuentra estresada por la 
alteración del sustrato (Adams et al., 2015), con la finalidad de que realicen la descomposición del contaminante al utilizarlo como fuente de carbono (Volke \& Velasco, 2002; Benavides et al., 2006; Pérez \& Saval, 2007; Vásquez et al., 2010; Adams et al., 2015); esta técnica aprovecha el hecho de que las bacterias son el grupo microbiano más versátil en cuanto a la biodegradación de hidrocarburos por su crecimiento y metabolismo rápido, plasticidad genética y su adaptabilidad a una gran variedad de ambientes (Pérez et al., 2008; Maldonado-Chávez et al., 2010).

Los microorganismos empleados se pueden obtener directamente de poblaciones naturales (Ortiz et al., 2006), previo a su aplicación se deben de realizar cultivos de enriquecimiento, aislar los microorganismos capaces de utilizar o co-metabolizar el contaminante y reproducirlos hasta obtener una gran cantidad de biomasa (Alexander, 1994; Volke \& Velasco, 2002). Estos microorganismos pueden proceder de diferentes fuentes como son las aguas residuales y el estiércol bovino (Devi et al., 2011), y cada microorganismo tiene diferentes actividades enzimáticas y preferencia por la degradación de ciertos compuestos. El éxito de esta técnica se basa en la habilidad de los microorganismos seleccionados para competir con los microorganismos autóctonos en el sitio contaminado, en la estructura del contaminante presente y su disponibilidad, el tamaño de la población microbiana y el ambiente físico (Adams et al., 2015). La bioaumentación y bioestimulación influyen notablemente sobre la degradación de lodos de petróleo (Tabla 3), sin embargo la combinación de ambas técnicas mejora la remoción de estos contaminantes del sustrato (Devi et al., 2011; Adams et al.,2015).

Tabla 3. Técnicas de biorremediación usadas con éxito en sustratos contaminados con hidrocarburos derivados del petróleo.

\begin{tabular}{|c|c|c|c|c|c|}
\hline Técnica & $\begin{array}{c}\text { Fuente de } \\
\text { Carbono }\end{array}$ & $\begin{array}{c}\text { Concentración } \\
\text { inicial de HTP } \\
\mathrm{mg} \mathrm{kg}^{-1}\end{array}$ & $\begin{array}{c}\% \\
\text { Degradación }\end{array}$ & $\begin{array}{c}\text { Tiempo } \\
\text { (días) }\end{array}$ & Referencia \\
\hline Bioaumentación & $\mathrm{PC}$ & 4,200 & 43 & 35 & $\begin{array}{c}\text { Chang et al., } \\
2011\end{array}$ \\
\hline Bioaumentación & Diesel & 16,300 & 83 & 120 & $\begin{array}{l}\text { Fan et al., } \\
2013\end{array}$ \\
\hline Bioaumentación & LA & 765,000 & 34 & 30 & $\begin{array}{c}\text { Devi et al., } \\
2011\end{array}$ \\
\hline Bioestimulación & HPC & ND & 100 & 20 & $\begin{array}{c}\text { Hamzah et al., } \\
2014\end{array}$ \\
\hline Bioestimulación & ALU & ND & 79 & 84 & $\begin{array}{c}\text { Abioye } e t \text { al., } \\
2012\end{array}$ \\
\hline Bioestimulación & LP & 334,766 & 30 & 15 & $\begin{array}{l}\text { Castorena- } \\
\text { Cortés et al., } \\
2009\end{array}$ \\
\hline Bioestimulación & LA & 765,000 & 23 & 30 & $\begin{array}{c}\text { Devi et al., } \\
2011\end{array}$ \\
\hline
\end{tabular}




\begin{tabular}{|c|c|c|c|c|c|}
\hline $\begin{array}{l}\text { Bioestimulación } \\
\text { Bioaumentación }\end{array}$ & LA & 765,000 & 44 & 30 & $\begin{array}{c}\text { Devi et al., } \\
2011\end{array}$ \\
\hline Bioestimulación & $\mathrm{FF}$ & 250 & 59 & 6.5 & $\begin{array}{c}\text { Kekacs et al., } \\
2015\end{array}$ \\
\hline Bioestimulación & $\mathrm{FF}$ & 968 & 55 & 14 & $\begin{array}{c}\text { Strong et al., } \\
2014\end{array}$ \\
\hline Fitorremediación & ALU & ND & 90 & 180 & $\begin{array}{l}\text { Agamuthu et } \\
\text { al., } 2010\end{array}$ \\
\hline Fitorremediación & $\mathrm{P}$ & 5,000 & 63 & 127 & $\begin{array}{c}\text { Peng et al., } \\
2009\end{array}$ \\
\hline Fitorremediación & PS & 48,800 & 35 & 60 & $\begin{array}{c}\text { Hutchinson et } \\
\text { al., } 2001\end{array}$ \\
\hline
\end{tabular}

\subsection{Fitorremediación}

La fitorremediación es una técnica utilizada para la recuperación de suelos contaminados con compuestos orgánicos (Frick et al., 1999, Peña-Castro et al., 2006; Maldonado-Chávez et al., 2010) y elementos potencialmente tóxicos (Raskin et al., 1996; Foroughbakch et al., 2004; Vassilev et al., 2004; Gutiérrez-Espinoza et al., 2011; Ruíz et al.,2013; SánchezLópez et al; 2015; González-Chávez et al., 2015), consiste en utilizar especies vegetales para recuperar de forma biológica el suelo, basándose en la capacidad que tienen de metabolizar o inmovilizar los componentes tóxicos mediante procesos de oxidación metabólica o co-oxidación (Pérez et al., 2002; Gutiérrez-Espinoza et al., 2011). Es una tecnología de bajo costo debido a que utiliza la energía solar como fuente de poder (Carpena \& Bernal, 2007; Ruíz et al., 2013); además es limpia y eficiente, ya que no utiliza reactivos químicos peligrosos, no afecta la estructura del suelo y la toxicidad remanente de los suelos recuperados es menor que en suelos tratados con otras tecnologías (Carpena \& Bernal, 2007; Peña-Castro et al., 2006; Ruíz et al., 2013). La fitorremediación es efectiva para el tratamiento de contaminantes como metales pesados, metales radioactivos, compuestos orgánicos y compuestos derivados del petróleo presentes en suelo, agua, aire o sedimentos (Delgadillo-López et al., 2011).

En la fitorremediación existe una interacción de la especie vegetal, la microbiota del suelo y la rizosfera y técnicas agronómicas que inactivan o degradan los elementos tóxicos (Fig. 3), transformándolos en compuestos orgánicos inocuos (Cunningham et al., 1996; Pérez et al., 2002; Vassilev et al., 2004; Garbisu et al., 2007; Thompson et al., 2008).

Los mecanismos de fitorremediación se pueden clasificar como rizodegradación, fitoextracción, fitodegradación y fitoestabilización (Van Deuren et al., 1997; Hutchinson et $a l ., 2011)$. En general el proceso de fitorremediación puede ocurrir de tres formas, en la primera la rizosfera mejora las propiedades físico-químicas de los suelos contaminados al favorecer la aireación e introducción de nutrimentos mediante la penetración radicular, favoreciendo la riqueza y diversidad microbiológica; la segunda consiste en la 
comunicación directa entre vegetal y microorganismo, en este caso la planta crea una zona favorable para el desarrollo selectivo de los microorganismos degradadores del contaminante, la tercer forma consiste en la degradación del contaminante por la planta mediante la extracción de agua hacia la parte aérea y la reversión del gradiente hidráulico que favorece la inmovilización o degradación del contaminante en la raíz y parte aérea mediante la producción de enzimas vegetales (Peña-Castro et al., 2006).

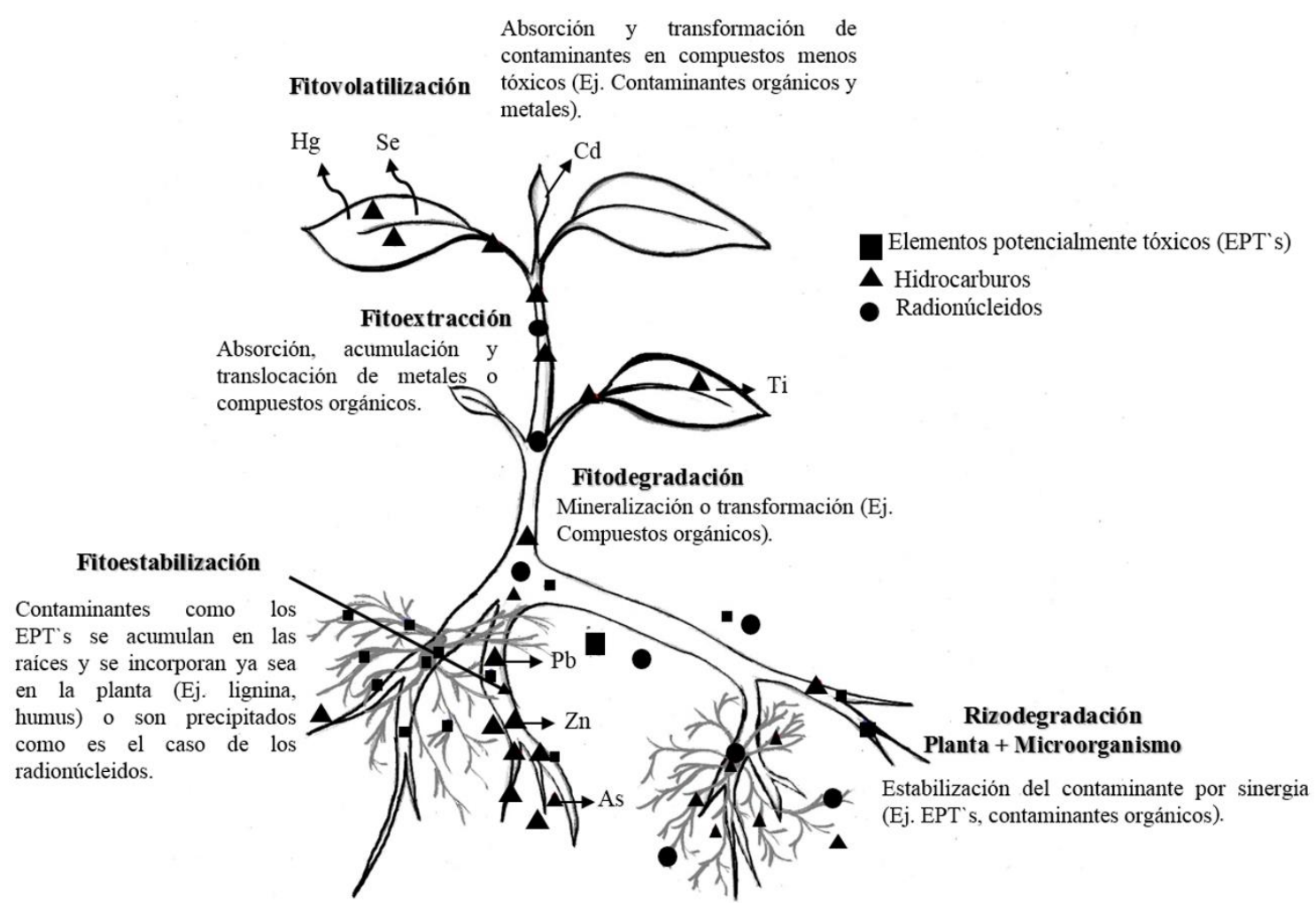

Fig. 3. Principales estrategias en fitorremediación. En el sistema suelo-plantamicroorganismo los elementos potencialmente tóxicos (cuadros), hidrocarburos (triángulos) y radionúcleidos (círculos) pueden ser transformados, metabolizados o acumulados en puntos específicos del sistema.

\section{TRANSFORMACIÓN DE LOS COMPUESTOS ORGÁNICOS EN LA BIORREMEDIACIÓN}

La biorremediación utiliza los microorganismos para reducir, eliminar, contener o transformar en compuestos inocuos los contaminantes (Adams et al., 2015), mediante la mineralización, transformación o alteración de los mismos (Shannon \& Unterman, 1993). Los microorganismos nativos del suelo juegan un rol importante en la biorremediación como agentes bioquímicos que transforman compuestos orgánicos complejos en compuestos inorgánicos simples o en sus constituyentes elementales mediante la mineralización (Adams et al., 2005); al disponer de capacidades metabólicas para adsorber 
las partículas del suelo por intercambio iónico, las cuales tienen carga negativa, las bacterias y el suelo pueden mantenerse unidas por enlaces iónicos que envuelven cationes polivalentes (Killham, 1994; Venosa et al., 2001), de tal forma que el proceso de biorremediación produce energía a través de una reacción redox que incluye la respiración y otras funciones biológicas necesarias para el mantenimiento y la reproducción de la célula microbiana, para que esto ocurra es necesario uno o más sistemas que provean una fuente de energía mediante un donador de electrones, un aceptor de electrones y nutrientes. En el proceso de biorremediación se pueden encontrar diferentes microorganismos aceptores de electrones oxígeno, nitrato, manganeso, hierro III, sulfato o un reductor de dióxido de carbono (Adams et al., 2015), mientras que el contaminante se encarga de proveer los electrones.

\section{CONCLUSIONES}

La explotación de gas natural mediante técnicas no convencionales va en aumento con el paso del tiempo, elevando de igual forma la cantidad de lodo residual del cual a nivel mundial se generaron en $2015141,500 \mathrm{~m}^{3}$. Las técnicas de biorremediación son una opción para recuperar estos sustratos. En particular la bioestimulación se ha utilizado para biorremediar lodos de perforación, removiendo hasta un 30\% de los HTP en un lapso de 15 días y en el caso de lodos y fluidos de perforación, se ha obtenido la remoción de hasta el $59 \%$ del carbono orgánico contenido en los fluidos de fractura en tiempos menores a 7 días. Este hecho representa una opción prometedora para el caso de México donde, dadas sus reservas estimadas de gas natural contenido en lutitas, se podrían generar hasta $1.09 \times 10^{8}$ $\mathrm{m}^{3}$ de lodos residuales de fractura hidráulica.

\section{AGRADECIMIENTOS}

Agradecemos a la Universidad Autónoma de Tamaulipas el apoyo otorgado a A. Maldonado Torres para la realización de una estancia de investigación en la Centro de Investigación en Ciencias Biológicas de la Universidad Autónoma de Tlaxcala.

\section{CONFLICTO DE INTERESES}

Los autores declaran que no existe conflicto de intereses.

\section{REFERENCIAS}

Abioye O., Agamuthu P. \& Abdul-Aziz A. 2012. Biodegradation of used lubricating oil by microbes isolated from pristine soil environment. Malaysian Journal of Science 31 (1): 1-7. 
Adams G., Tawari-Fufeyin P. \& Igelenyah E. 2014. Bioremediation of spent oil contaminated soils using poultry litter. Research Journal in Engineering and Applied Sciences 3 (2): 124-130.

Adams G., Fufeyin P., Okoro S. \& Ehinomen I. 2015. Bioremediation, Biostimulation and Bioaugmention: A Review. International Journal of Environmental Bioremediation \& Biodegradation 3 (1): 28-39.

Adams R., Zavala-Cruz, J. \& Morales-García F. 2008. Concentración residual de hidrocarburos en el suelo del trópico II: afectación a la fertilidad y su recuperación. Interciencia 33 (7): 483-489.

Agamuthu P., Abioye O. \& Abdul A. 2010.Phytoremediation of soil contaminated with used c lubricating oil using Jatropha curcas. Journal of Hazardous Materials 179 (1): 891894.

Alexander M. 1994. Biodegradation and bioremediation. Academic Press. USA. pp 302.

Al-Saleh E., Drobiova H.\& Obuekwe C. 2009. Predominant culturable crude oil-degrading bacteria in the coast of Kuwait. International Biodeterioration \& Biodegradation 63 (4): 400-406.

Atlas R. \& Bartha R. 1988. Microbial Ecology: Fundamentals and Applications. Benjamin Cummings. California. USA. pp 533.

Benavides M., Quintero G., Guevara A., Jaimes C., Gutiérrez S. \& Miranda J. 2006. Biorremediación de suelos contaminados con hidrocarburos derivados del petróleo. NOVA Publicación Científica en Ciencias Biomédicas 4 (5): 1-116.

Bewley J., Alexander G. \& Webb G. 2001. Ex situ and In situ bioremediation of former oil distribution. Land Contamination and Reclamation 9: 1-15.

Bjørlykke K. 2010. Unconventional hydrocarbons: oil shales, heavy oil, tar sands, shale gas and gas hydrates. In: Petroleum Geoscience. Bjørlykke K. Springer. Berlin. Germany. pp 581-590.

Bordenave M., Goñi-Urriza S., Caumete P. \& Duran R. 2007. Effects of heavy fuel on the bacterial community structure of a pristine microbial mat. Applied and Environmental Microbiology 73 (19): 6089-6097.

Bundy J., Paten G. I. \& Campbel C. 2002. Microbial communities in different soil types do not converge after diesel contamination. Applied Microbiology 92 (2):276-288.

Carpena R. \&.M. Bernal. 2007. Claves de la fitorremediación: fitotecnologías para la recuperación de suelos. Ecosistemas 16 (2): 1-3.

Castorena-Cortés G., Roldán-Castillo T., Zapata-Peñasco I., Reyes-Ávila J., Quej-Aké L., Marín-Cruz J. \& Olguín-Lora P. 2009. Microcosm assays and Taguchi experimental design for treatment of soil sludge containing high concentration of hydrocarbons. Bioresourse Technology 100 (23): 5671-5677. 
Chang K., Ibrahim D. \& Ibrahim C. 2011. A laboratory scale bioremediation of Tapis crude oil contaminated soil by bioaugmentation of Acinetobacterbaumannii T30C. African Journal of Microbiology Research 5 (18): 2609-2615.

Chen H., He M., Han B., Li Z. \& Li P. 2012. Research of shale gas in China. In: Du W. (Ed). 2012. Informatics and Management Science I. Springer. England 259-263 p.

Chen Z., Osadetz K. \& Chen X. 2015. Economic appraisal of shale gas resources, an example from the Horn River shale gas play, Canada. Petroleum Science 12 (4): 712-725.

Cunningham S., Anderson T., Schwab A. \& Hsu F. 1996. Phytoremediation of soils contaminated with organic pollutants. Advances in Agronomy 56 (4):55-114.

Delgadillo-López A., González-Ramírez C., Prieto-García F., Villagómez-Ibarra J. \& Acevedo-Sandoval O. 2011. Fitorremediación: una alternativa para eliminar la contaminación. Tropical and Subtropical Agroecosystems14 (2): 597-612.

Devi M., Reddy M., Juwarkar A., Sarma, P. \& Mohan S. 2011. Effect of co-culture and nutrients supplementation on bioremediation of crude petroleum sludge. Clean-Soil, Air, Water 39 (10): 900-907.

Environmental Protection Agency. 2015. TENORM: Oil and Gas Production Wastes. In: http://www.epa.gov/radiation/tenorm-oil-and-gas-production-wastes

Fair J. 2014. Radionucléotidos en las aguas Residuales de la Fracturación Hidráulica Manejo de una Mezcla Tóxica. Salud Pública de México 56 (4): 408-415.

Fan M., Xie R. \& Qin G. 2014. Bioremediation of petroleum-contaminated soil by a combined system of biostimulation-bioaugmentation with yeast. Environmental Technology 35 (4): 391-399.

Foroughbakhch R., Céspedes A., Alvarado M., Núñez A. \& Badii M. 2004. Aspectos ecológicos de los manglares y su potencial como fitorremediadores en el Golfo de México. Ciencia UANL 7 (2): 203-208.

Frick C., Farrell R. \& Germida J. 1999. Assessment of phytoremediation as an in situ technique for cleaning oil-contaminated sites. Department of Soil Science University of Saskatchewan. Saskatchewan Canada. pp 88.

Garbisu C., Becerril M., Epelde L. \& Alkorta I. 2007. Bioindicadores de la calidad del suelo: herramienta metodológica para la evaluación de la eficacia de un proceso fitorremediador. Ecosistemas 16 (2): 1-6.

Gonçalves M., Machado S., Marques M. \& Vidal D. 2015. Emission of volatile organic compounds and greenhouse gases from the aerobic Bioremediation of soils contaminated with diesel. Water, Air, Soil Pollution 226 (1): 50-59.

González-Chávez M., Sánchez-López A. \& Carrillo-González R. 2015. Arsenic concentration in wild plants growing on two mine tailings. Pharmacognosy Communications 5 (3):1-11 
Gregory K., Vidic R. \& Dzombak D. 2011. Global water sustainability: water management challenges associated with the production of shale gas by hydraulic fracturing. Elements 7 (3): 181-186.

Ground Water Protection Council and Interstate Oil and Gas Compact Comission (GWPC \& IOGCC). 2015. What Chemical are used. Frac Focus Chemical Disclosure Registry. In: fracfocus.org/chemical-use/what-chemical-are-used

Gutiérrez-Espinoza R., Melgoza-Castillo A., Alarcón-Herrera M., Ortega-Gutiérrez J. Prado-Tarango D. \& Cedillo-Alcantar M. 2011. Germinación del girasol silvestre (Helianthus annuus L.) en presencia de diferentes concentraciones de metales. Revista Latinoamericana de Biotecnología Ambiental y Algal 2 (1): 49-56.

Hart W. 2013. Impacto del desarrollo del shale gas en el suministro de líquidos de gas natural $y$ en las materias primas petroquímicas. http://www.imiq.org/wpcontent/uploads/2013/07/IMPACTO-DEL-SHALE-GAS.pdf

Hamzah A., Chia-Wei P., Pek-Hoon Y. \& Nurul H. 2014. Oil Palm Empty Fruit Bunch and Sugarcane Bagasse Enhance the Bioremediation of Soil Artificially Polluted by Crude Oil. Soil and Sediment Contamination: An International Journal 23 (7): 751-762.

Hernández A., Ferrera-Cerrato R. y Rodríguez R. 2003. Bacterias de vida libre fijadoras de nitrógeno atmosférico en rizosfera de frijol contaminada con queroseno. TERRA Latinoamericana 21 (1): 81-89.

Hutchinson S., Banks M. \& Schwab A. 2001. Phytoremediation at aged petroleum sludge: effect of inorganic fertilizer. Journal of Environmental Quality 30 (2): 395-403.

International Centre for Soil and Contaminated Sites (ICSS).2006. Manual for biological remediation techniques. ICSS at the German Federal Environmental Agency. Dessau Germany $81 \mathrm{pp}$.

International Energy Agency (IEA). 2012. Golden rules for a golden age of gas. World Energy Outlook Special Report on Unconventional Gas. IEA. France. pp.143.

International Energy Agency (IEA). 2013. Technically recoverable shale oil and shale gas resources: an assessment of 137 shale formations in 41 countries outside the United States. World Energy Outlook Special Report on Unconventional Gas. IEA. U.S.. pp.730.

Jiang, M., Hendrickson, C. T. \& VanBriesen, J. M.2014. Life cycle water consumption and wastewater generation impacts of a Marcellus Shale Gas Well. Environmental Science and Technology 48 (1): 1911-1920.

Kekacs, D., Drollete, B. D., Brooker, M., Plata, D. L. \& Mouser, P. J. 2015. Aerobic biodegradation of organic compounds in hydraulic fracturing fluids. Biodegradation 26 (1): 271-287.

Killham K. 1994. Soil Ecology. Cambridge University Press. Cambridge UK. pp.264.

Leahy J. \& Colvwell R. 1990. Microbial Degradation of Hidrocarbons in the Environment. Microbial Reviews 53 (3): 305-315. 
Ma F., Wang Y. \& Wang L. 2013. Enhancing shale gas recovery by high temperature supercritical CO2 flooding. Chemistry and Technology of Fuels and Oils 49 (4): 348-354.

Maldonado-Chávez E., Rivera-Cruz M., Izquierdo-Reyes F. \& Palma-López D. 2010. Efectos de rizosfera, microrganismos y fertilización en la biorremediación y fitorremediación de suelos con petróleos crudo nuevo e intemperizado. Universidad y Ciencia 26 (2): 121-136.

Margesin R. \& Schiner F. 2001. Bioremediation (natural attenuation and biostimulation) of diesel-oil-contaminated soil in an alpine glacier skiing area. Applied Environmental. Microbiology 67 (7): 3127-3133.

Marín J., Moreno J., Hernández T. \& García C., 2006. Bioremediation by composting of heavy oil refinery sludge in semiarid conditions. Biodegradation 17 (3): 251-261.

Moreu E. 2013. Marco jurídico de la extracción de hidrocarburos mediante fractura hidráulica (Fracking). Revista Catalana de Dret Ambiental 3 (2): 1-43.

Moubasher H., Hegazy A., Mohamed N., Moustafa Y., Kabiel H. \& Hamad A. 2015. Phytoremediation of soils polluted with crude petroleum oil using Bassia scoparia and its associated rhizosphere microorganism. International Biodeterioration and Biodegradation 98 (3): 113-120.

Mrayyan B. \& Battikhi.M. 2005. Biodegradation of total organic carbon (TOC) in Jordanian petroleum sludge. Journal of Hazardous Materials 120(1-3): 127-134.

Mukherjee A. \& Das K. 2005. Correlation between diverse cyclic lipopeptides production and regulation of growth and substrate utilization by Bacillus subtilisstrains in a particular habitat. FEMS Microbiology Ecology 54 (3): 479-489.

Ning X., Wenxiang W., Pingfang H. \& Xiaoping L. 2009. Effects of ultrasound on oily sludge deoling. Journal of Hazardous Materials 171 (1): 914-917.

Olguín E., Hernández M. \& Sánchez G. 2007. Contaminación de manglares por hidrocarburos y estrategias de biorremediación, fitorremediación y restauración. Revista Internacional de Contaminación Ambiental 23 (3): 139-154.

Ortíz I., Sanz J., Dorado M. \& Villar S. 2006. Técnicas de recuperación de suelos contaminados. Informe de vigilancia tecnológica. Universidad de Alcalá. Alcalá España. pp 108

Ouyang W., Liu H., Murygina V., Yu Y., Xiu Z. \& Kalyuzhnyi S., 2005. Comparison of bioaugmentation and composting for remediation of oily sludge: a field-scale study in China. Process Biochemistry 40 (1): 3763-3768

Peng S., Zhou Q., Cai Z. \& Zhang A. 2009. Phytoremediation of petroleum contaminated soils by Mirabilis jalapa L. in a greenhouse plot experiment. Journal of Hazardousmaterials168 (2): 1590-1496.

Peña-Castro J., Barrera-Figueroa B., Ruiz-Medrano R. \& Xoconostle-Cazares B. 2006. Bases moleculares de la fitorremediación de hidrocarburos totales del petróleo. TERRA Latinoamericana 24 (4): 529-539. 
Pérez J., García G. \& Esparza F. 2002. Papel ecológico de la flora rizosférico en fitorremediación. Avance y Perspectiva 21 (1): 297-300.

Pérez N. \& Saval S. 2007. Biorremediación en biopilas a nivel piloto de un suelo contaminado con diesel mediante bioestimulación, bioaumentación y adición de composta vegetal. In: XII Congreso Nacional de Biotecnología y Bioingeniería. UAM-Iztapalapa. México.http://www.smbb.com.mx/congresos\%20smbb/morelia07/TRABAJOS/Area_IV/C ARTELES/CIV-16.pdf

Pérez R., Camacho M., Gómez J., Avalos A. \& Cantero D. 2008. Aislamiento y selección de una cepa bacteriana degradadora de hidrocarbur81os a partir de suelos contaminados con petróleo. Revista CENIC. Ciencias Biológicas 39 (1): 44-51.

Perfumo A., Banat I. Marchant R. \& Vezzulli L. 2007. Thermally enhanced approaches for bioremediation of hydrocarbon-contaminated soils. Chemosphere 66 (1): 179-184.

Raskin I. 1996. Plant genetic engineering may help with environmental cleanup. Proceedings of the National Academy of Sciences of the United States of America. U.S.A. 93 (8): 3164-3166.

Rojas-Avelizapa N., Roldán-Carrillo T., Zegarra-Martínez H., Muñoz-Colunga A. \& Fernández-Linares L. 2007. A field trial and ex-situ bioremediation of a drilling mudpolluted site. Chemosphere 66 (9): 1595-1600.

Ruíz A., Carrillo-González R., González-Chávez M. \& Soto R. 2013. Potential of castor oil (Ricinus communis L.) for phytoremediation of the mine tailing and oil production. Journal of Environmental Management 114 (1): 316-323.

Ryzhov A., Avakyan T., Smolenski E. \& Lapidus A. 2012. Dependence of the hydrogen content of oil shale gas on the oil shale gasification conditions. Docklady Chemistry 447 (1): 233-237.

Sabate D., Carrillo L. \& Audisio M. 2009. Inhibition of Paenibacillus larvae and Ascosphaera apis by Bacillus subtilis isolated from honey bee gut and honey samples. Research in Microbiology 160 (3): 193-199.

Sánchez-López A., González-Chávez M., Carrillo-González R., Vangrosveld J. \& DíazGarduño M. 2015. Wild Flora of mine tailings: perspectives for use in phytoremediation of potencially toxic elements in a semi-arid region in Mexico. International Journal of Phytoremediation 17 (5): 476-484.

Secretaría de Energía 2014. Anuario Estadístico Petróleos Mexicanos Prospectiva de Gas Natural y Gas LP 2014-2028. SENER. México. pp 20.

Secretaría de Medio Ambiente y Recursos Naturales (SEMARNAT). 2006. NOM-052SEMARNAT-2006. Norma Oficial Mexicana que establece las características, el procedimiento de identificación, clasificación y los listados de residuos peligrosos. SEMARNAT. México. pp 32.

Semple K., Reid B.\& Fermor T. 2001. Impact of composting strategies on the treatment of soils contaminated with organic pollutants. Environmental Pollution 112 (2): 269-283. 
Shannon M.\& Unterman R. 1993. Evaluating bioremediation: distinguishing fact from fiction. Annual Review of Microbiology 47 (1): 715-724.

Strong, L. C., Gould, T., Kasinkas, L., Sadowsky, M. J., Aksan, A. \& Wackett, P. 2013. Biodegradation in waters from hydraulic fracturing: chemistry, microbiology and engineering. Journal of Environmental Engineering 140 (5): B4013001

Thompson O., Wolf D., Mattice J.\& Thoma G. 2008. Influence of nitrogen addition and plant root parameters on phytorremediation of pyrene-contaminated soil. Water Air Solution Pollution 189 (1-4): 37-47

U.S. Energy Information Administration.2013. Technically recoverable shale oil and shale gas resources: an assessment of 137 shale formations in 41 countries outside the United States. U.S. Energy Information Administration. U.S.S. pp. 730 https://www.eia.gov/analysis/studies/worldshalegas/

USEPA 2012. Mine Waste Technology Program. Project 12: sulfate reducing bacteria reactive wall demonstration. Final Report. In: http:// www.epa.gov/ORD/NRMRL/std/mtb/mtbdocs/actiiiproj12.pdf; 2002.

Van Deuren J., Wang Z.\& Ledbetter J. 1997. Remediation Technologies Screening Matrix and Reference Guide. Technology Innovation. EPA. In:http://www.epa.gov/tio/remed.htm.

Vásquez M., Guerrero J. \& Quintero A. 2010. Biorremediación de lodos contaminados con aceites lubricantes usados. Revista Colombiana de Biotecnología 12 (1): 141-157.

Vassilev A., Schwitzguébel J., Thewya T., Van der Lelie D. \& Vangronsveld J. 2004. The use of plants for remediation of metal-contaminated soils. The Scientific World Journal 4 (1): 9-34

Venosa A., Lee K., Suidan M., Garcia-Blanco S., Cobanli S., Moteleb M., Haines J., Tremblay G. \& Hazelwood M. 2002. Bioremediation and biorestoration of a crude oil contaminated freshwater wetland on the St. Lawrence River. Bioremediation Journal 6 (3): 261-281.

Volke T. \& J. Velasco. 2002. Tecnologías de remediación para suelos contaminados. INESEMARNAT. México. pp 62.

Wang W., Yao J., Sun H. \& Song W. 2015. Influence of gas transport mechanism of the productivity of multi-stage fractured horizontal wells in shale gas reservoirs. Petroleum Sciences 12 (4): 664-673.

Wei M., Duan Y., Fang Q., Wang R., Yu B. and Yu C. 2013. Mechanism model for shale gas transport considering diffusion, adsorption/desorption and Darcy flow. Journal Center South University 20: 1928-1937.

Wheeler D., MacGregor M., Atherton F., Christmas K., Dalton S., Dusseault M., Gagnon G., Hayes B., MacIntosh C., Mauro I. \& Ritcey R. 2015. Hydraulic fracturing-integrating public participation with an independent review of the risks and benefits. Energy Policy 85 (1): 299-308. 
Zagrean C. 2002. Oil Exploration and Production Wastes Initiative. Department of Toxic Substances Control Hazardous Waste Management Program Statewide Compilance Division. USA. pp 55.

Zhang X., Sun Y. \& Duncan I. 2016. Shale gas wastewater management under uncertainty. Journal of Environmental Management 165 (1): 188-198. 